\title{
Islands Pflanzenwelt zwischen Feuer und Eis, Teil 1: Geschichte und Besonderheit der Pflanzenwelt Islands
}

\author{
Karin Steinecke \& Hilke Steinecke
}

\begin{abstract}
Iceland is geologically young. The island is well known for its active vulcanism and large glaciers and today natural forests are nearly absent. Since the settlement by Norwegian Vikings more than 1100 years ago, the natural forests have been destroyed. Geography, geology and history of vegetation are summarized.
\end{abstract}

\section{Zusammenfassung}

Das geologisch junge Island ist durch aktiven Vulkanismus geprägt. Daneben überziehen riesige Gletscher Island. Wälder gibt es heute fast nicht mehr. Seit dem Beginn der dauerhaften Besiedlung Islands durch norwegische Wikinger vor über 1100 Jahren wurden die ursprünglichen Birkenwälder kontinuierlich zerstört. Geographie, Geologie und Vegetationsgeschichte Islands werden vorgestellt.

\section{Geographie und Geologie Islands - ein kurzer Überblick}

Island nimmt eine Fläche von $103000 \mathrm{~km}^{2}$ ein, was etwa der Fläche von Bayern und BadenWürttemberg zusammen entspricht. Das administrativ zu Europa zählende Island ist die zweitgrößte Insel Europas und gleichzeitig die größte Insel vulkanischen Ursprungs der Erde. Island befindet sich in extremer Insellage im Nordatlantik knapp südlich des nördlichen Polarkreises. Der nördlichste Punkt der Hauptinsel liegt bei $66^{\circ} 32^{\prime} 29^{\prime \prime} \mathrm{N}$, der südlichste bei $63^{\circ} 23^{\prime} 25^{\prime \prime} \mathrm{N}$. Außerhalb dieser Grenzen existieren einige unbewohnte vorgelagerte Inseln und Vogelklippen, wobei die knapp nördlich des Polarkreises liegende Insel Kolbeinsey den nördlichsten Punkt Islands darstellt. Die erst 1963 bei einem zunächst unterseeischen Vulkanausbruch entstandene Insel Surtsey bildet den südlichsten Punkt Islands. Die kürzesten Distanzen Islands betragen zu Grönland $287 \mathrm{~km}$, zu den FäröerInseln $435 \mathrm{~km}$, zu Schottland $812 \mathrm{~km}$ und $\mathrm{zu}$ Norwegen $970 \mathrm{~km}$ (Nordal \& KRISTONSSON 1996). Rund $25 \%$ der Landesfläche liegen in Küstennähe zwischen 0 und 200 m (Siedlungsgrenze), knapp $40 \%$ der Fläche Islands befinden sich in einer Höhenlage von über $600 \mathrm{~m}$. Diese unbesiedelten Regionen sind vornehmlich im Inselinneren, dem sog. Hochland, anzutreffen. Mit 2119 m Höhe ist der Hvannadalshnúkur der höchste Berg Islands. Die meisten der markanten und höheren (Vulkan-)Berge erreichen
Höhen von rund 1500 m. Rund $93 \%$ der Gesamtbevölkerung Islands (2008: 310 000) wohnen überwiegend im Großraum Reykjavík.

Island gilt als der größte überseeische Bestandteil des Mittelatlantischen Rückens, einem submarinen vulkanischen Höhenzug. An dieser Nahtstelle zwischen amerikanischer und eurasischer Kontinentalplatte steigt seit der Öffnung des Nordatlantiks im Jura heißes Mantelgestein auf und drückt die beiden Platten mit einer Geschwindigkeit von heute noch etwa $1-2 \mathrm{~cm}$ pro Jahr auseinander. An anderer Stelle entstanden auf dem Mittelatlantischen Rücken durch Vulkanismus z. B. die Azoren sowie die nördlich von Island liegende Insel Jan Mayen. Gemäß seiner Entstehung befinden sich die ältesten Gesteine Islands im äußersten Westen und Osten der Insel. Mit einem maximalen Alter von 17 Mio. Jahren sind sie aber im Vergleich zu den meisten Gesteinen Festlandeuropas geologisch sehr jung (GUDMUNDSsON \& Kjartansson 1996). Noch jünger sind die Gesteine der aktiven Vulkanzone Islands, die sich mitten durch Island zieht. Der aktive Vulkanismus Islands äußert sich in Form von immer wieder auftretenden Eruptionen (durchschnittlich etwa alle 4-5 Jahre) der etwa 30 heute noch aktiven Vulkane, häufigen Erdbeben und der Existenz von Geothermalgebieten (z. B. Gudmundsson 1984).

Die geothermale Energie wird in Island gewerblich (Beheizung von Gehwegen, Schwimm- 


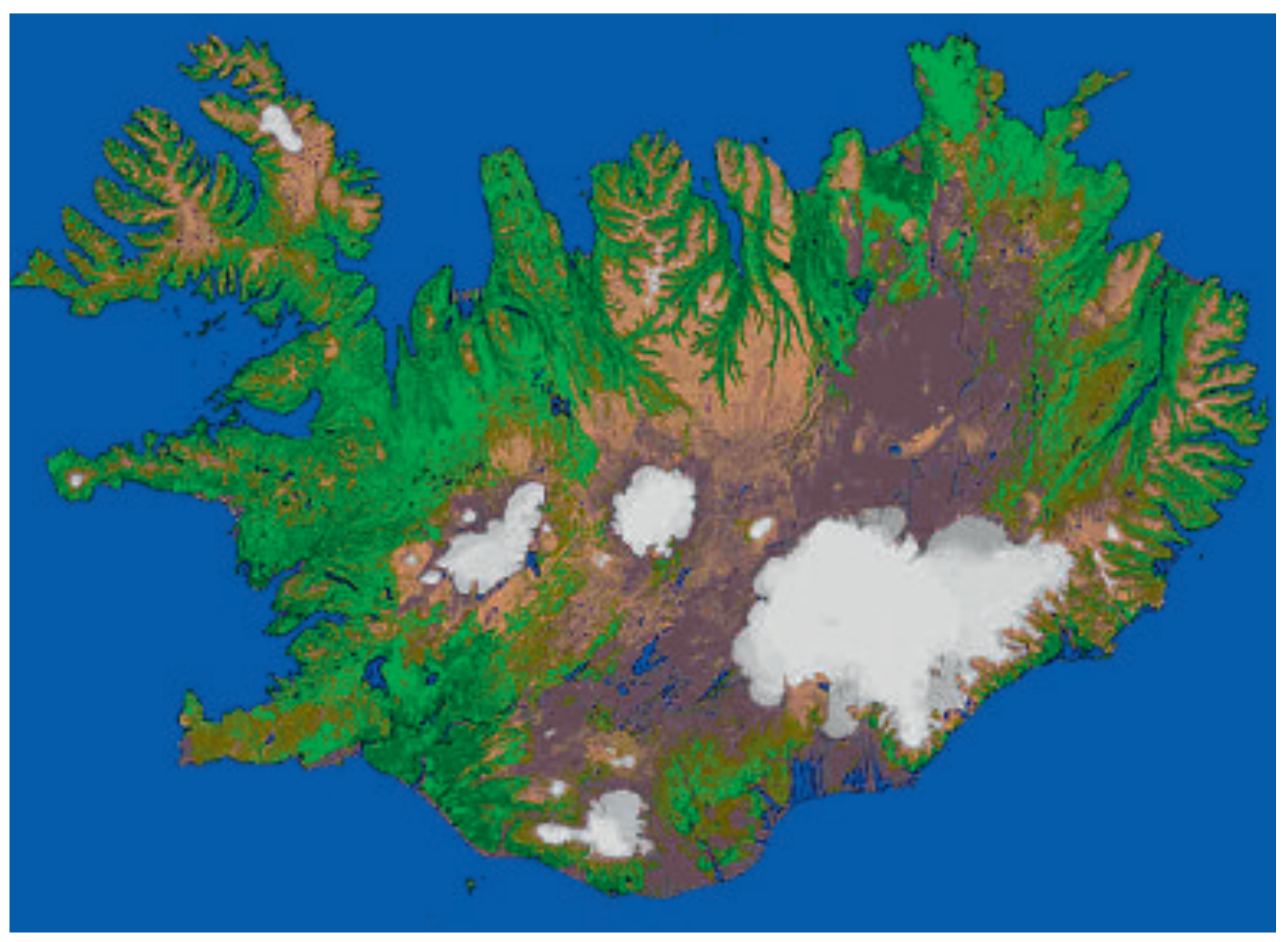

bädern, Aquakulturen z. B. von Lachs sowie von Gewächshäusern z. B. für den Anbau von Tomaten, Gurken, Champignons, Schnittblumen und sogar Bananen) oder industriell (Stromerzeugung über Dampfturbinen, Betreibung eines Fernheizsystems, bis vor wenigen Jahren auch Gewinnung von Kieselgur und Meersalz) genutzt. Als Teil des Mittelatlantischen Rückens besteht Island zu 90\% aus vulkanischen Gesteinen, dazu kommen marine und terrestrische Sedimente. Die Erstarrungsgesteine wiederum sind zu $80 \%$ basisch mit einem geringen Silikatgehalt unter 54\%. Demzufolge sind auch die Feinerdeböden verhältnismäßig nährstoff- und basenreich, was der Vegetation zugute kommt. Allerdings sind die Böden Islands vielerorts aufgrund ihrer Entstehung aus vulkanischem Ausgangsmaterial sehr locker und wenig bindig und damit stark

Abb. 1: Übersichtskarte von Island. Die weißen Bereiche markieren die riesigen Gletscher. (wind)erosionsgefährdet sowie gleichzeitig sehr wasserdurchlässig. So sind die nahezu vegetationsfreien Bereiche im Hochland als edaphische Wüsten anzusprechen, in denen die Bodentrockenheit - nicht der Niederschlagsmangel zum limitierenden Faktor für eine geschlossene Pflanzendecke wird.

Das Klima Islands ist überwiegend feuchtkaltgemäßigt, mit jährlichen Niederschlagssummen teilweise bis zu $1500 \mathrm{~mm}$ an der Südküste. Diese hohen Niederschlagssummen sind eine der Voraussetzungen für das Entstehen der dortigen großen Gletscher. Aufgrund der maritimen Lage ist das Klima Islands verständlicherweise in den Küstengebieten hochozeanisch, im Hochland deutlich kontinentaler geprägt. Der Süden, Westen und Nordwesten Islands werden vom warmen Golfstrom (Irminger-Strom) umströmt, im Norden und Nordosten führt der Ostgrönlandstrom (OstislandStrom) kaltes polares Oberflächenwasser herbei, der die dortigen Küsten in kalten Wintern schon von Packeis einschließen ließ und schon 

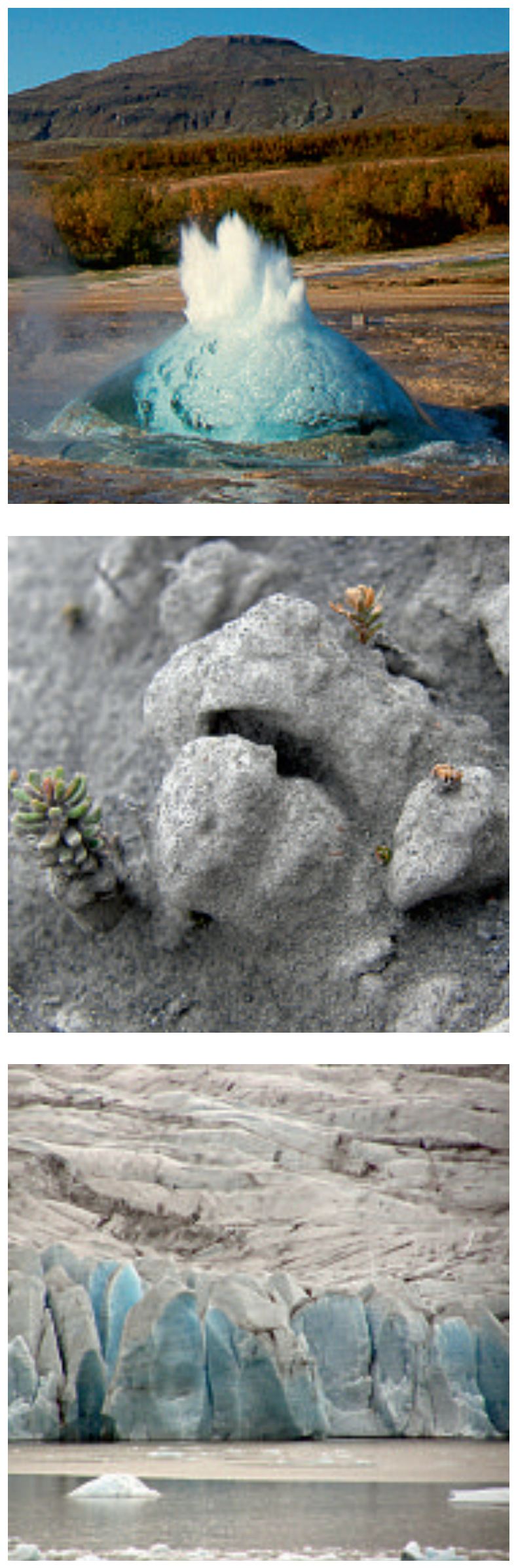

so manchen Eisbären auf einer Eisscholle von Grönland aus nach Island brachte. Für die im Südwesten küstennah liegende Hauptstadt Reykjavík beträgt die Jahresmitteltemperatur $4,3^{\circ} \mathrm{C}$, das Januarmittel $-0,5^{\circ} \mathrm{C}$ und das Julimittel 10,6 $6^{\circ} \mathrm{C}$ (Erhebungszeitraum: 1961-1990). Charakteristisch für Island sind neben den hohen Niederschlägen, den milden Wintern und kühlen Sommern auch die durch die zahlreichen hindurchziehenden dynamischen Tiefdruckgebiete (Island-Tief) verursachte Wechselhaftigkeit des Wetters sowie die zumeist sehr hohen Windgeschwindigkeiten, die ebenfalls großen Einfluss auf die Vegetation haben (Schmidt 1991, Schutzbach 1999).

\section{Flora und Vegetation}

Angesichts der außergewöhnlichen geologischen und geomorphologischen Landschaftselemente und einer gleichzeitig sehr vielfältigen Vogelwelt u.a. mit großen Beständen an Papageientauchern und anderen Seevögeln wird die Pflanzenwelt in der kargen Landschaft Islands von den meisten Besuchern kaum bewusst wahrgenommen. Die vielen Grüntöne sind insbesondere auf die vielen Moose zurückzuführen, die Pionierpflanzen auf jungen Lavafeldern darstellen. Dazu kommen noch einmal etwa 700 Flechtenarten, die den nackten Fels in vielen Farben schmücken. Besonders beeindruckende Beispiele des reichen KryptogamenWuchses auf Island sind Jahrhunderte alte Lavafelder (z. B. auf der Halbinsel Reykjanes nahe Reykjavík). Die Block- oder Stricklava ist dort von einer bei Trockenheit silberig grau und bei Nässe satt grün wirkenden, aber höchst trittempfindlichen Moosschicht aus Zackenmützenmoos (Racomitrium lanuginosum und andere Arten dieser Gattung) überzogen. Bach-

Abb. 2 (oben): Der Heißwassergeysir Strokkur baut gerade seine Fontäne auf.

Abb. 3 (Mitte): Der Ausbruch des Vulkans Eyjafjallajökull im April 2010 bedeckte in seiner Umgebung die Vegetation mit einer dichten Ascheschicht.

Abb. 4 (unten): Mitunter gewaltige Eismassen prägen das Land und beeinflussen die Pflanzenwelt. 
läufe und Quellfluren leuchten weithin in der Landschaft durch das Giftgrün des Pohl- oder Fadenmooses Pohlia wahlenbergii. Besonders farbintensive bunte Flecken in der Landschaft bilden beispielsweise auch Massenbestände des intensiv rosa blühenden Arktischen Thymians (Thymus praecox subsp. arcticus) oder des violett blühenden Wald-Storchschnabels (Geranium sylvaticum), der in Resten des ursprünglich subpolaren Birkenwaldes in geschützten Schluchten oder im Nationalpark Skaftafell oft massenhaft auftritt.

\subsection{Zusammensetzung und Eigenschaften der isländischen Flora}

Individuenreiche Massenbestände mancher Blütenpflanzen auf Island dürfen nicht darüber hinweg täuschen, dass die Flora wie auch die Fauna vergleichsweise artenarm ist (Dierssen 1996). Auf Island gibt es nur knapp 500 Arten heimischer Gefäßpflanzen (EINARsson 2005). Zum Vergleich: In Kanada sind etwa 3700 Arten heimisch (Government of Canada 1996), in Deutschland sind es etwa 2700 (KüHN \& KLOtz 2002), und in Norwegen etwa 1800 heimische Arten. Der Grund für diese relative Artenarmut liegt nicht vordergründig in den z. T. klimatisch sehr rauen und scheinbar unwirtlichen Lebensräumen Islands, sondern lässt sich vielmehr mit der nahezu vollständigen Vereisung Islands während der Eiszeiten und der nachfolgenden aufgrund der isolierten Lage im Nordatlantik erschwerten Wiederbesiedlung der vergleichsweise kleinen Insel erklären. Allerdings ist davon auszugehen, dass durch die Vergletscherung nicht alle Pflanzensippen vollständig ausstarben. Pollenanalysen in Proben alter isländischer Moore und Untersuchungen der heutigen Arealtypen verschiedener isländischer Pflanzenarten haben ergeben, dass etwa die Hälfte der heute vorkommenden Arten die Eiszeiten auf reliefbedingt eisfreien Gebirgsgipfeln sowie in einigen kleineren eisfreien Arealen entlang der Küsten überleben konnten (BucK-

Abb. 5: Bei Thingvellir driften die eurasische und die amerikanische Kontinentalplatten stetig auseinander.

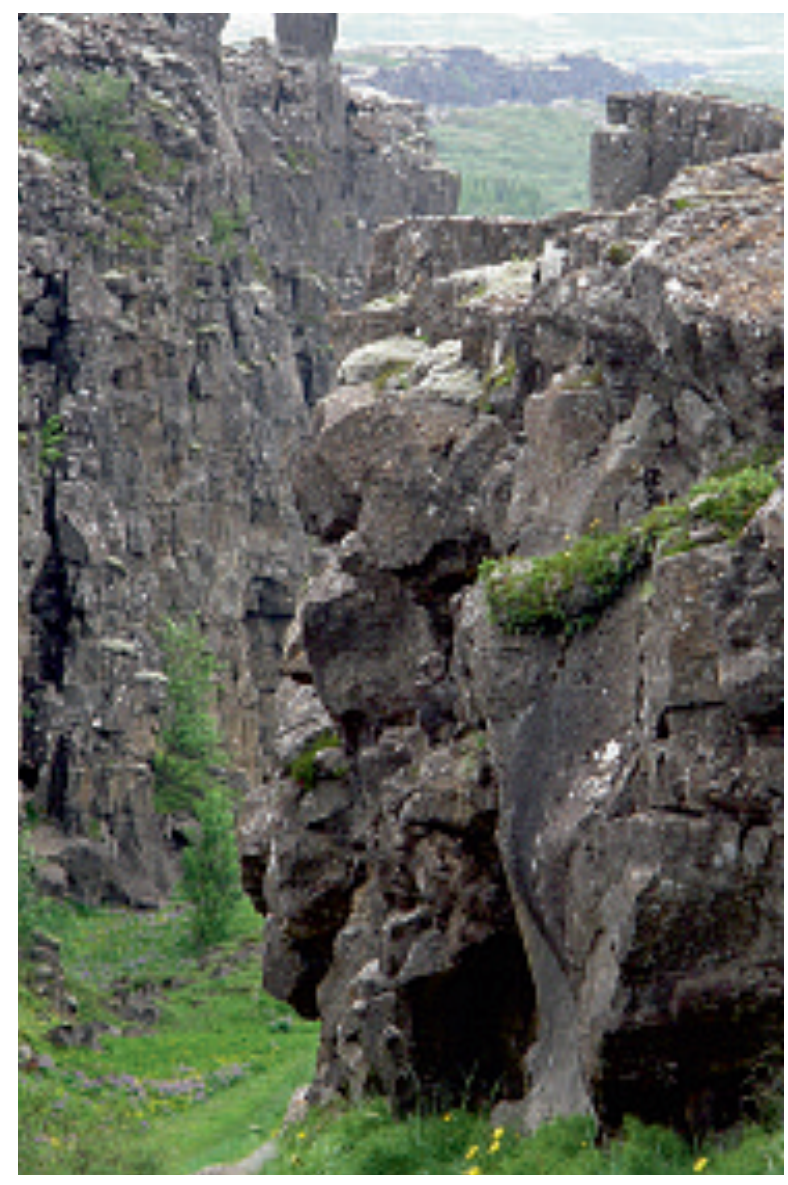

LANd \& Dugmore 1991). Die heutigen Verbreitungsgebiete vieler Arten (z. B. des Gamander-Ehrenpreises, Veronica chamaedrys) decken sich sehr gut mit der Lage der nachweislich eisfreien Bereiche in Island. Ein Beispiel für die Bedeutung glazialer Refugien gibt das heute wie früher unvergletscherte Bergmassiv Esjufjöll inmitten des Vatnajökull. Etwa 80 Arten höherer Pflanzen lassen sich heute in diesem Areal finden, das in alle Himmelsrichtungen mindestens $20 \mathrm{~km}$ durch vollkommen vergletscherte Bereiche von den nächsten von Pflanzen besiedelbaren Flächen getrennt ist. Fast alle der 45, heute auf der Roten Liste der geschützten Gefäßpflanzen Islands stehenden, aber nicht notwendigerweise geschützten Pflanzenarten, sind nicht durch menschliche Aktivitäten in ihrem Bestand bedroht, sie nehmen als Glazialrelikte ohnehin nur sehr kleine Areale auf Island ein (The Icelandic Institute of Natural History 2010). Zu den extrem seltenen Arten auf Island, die teilweise nur an einem einzigen Ort zu finden sind, gehören beispielsweise der Braunstie- 

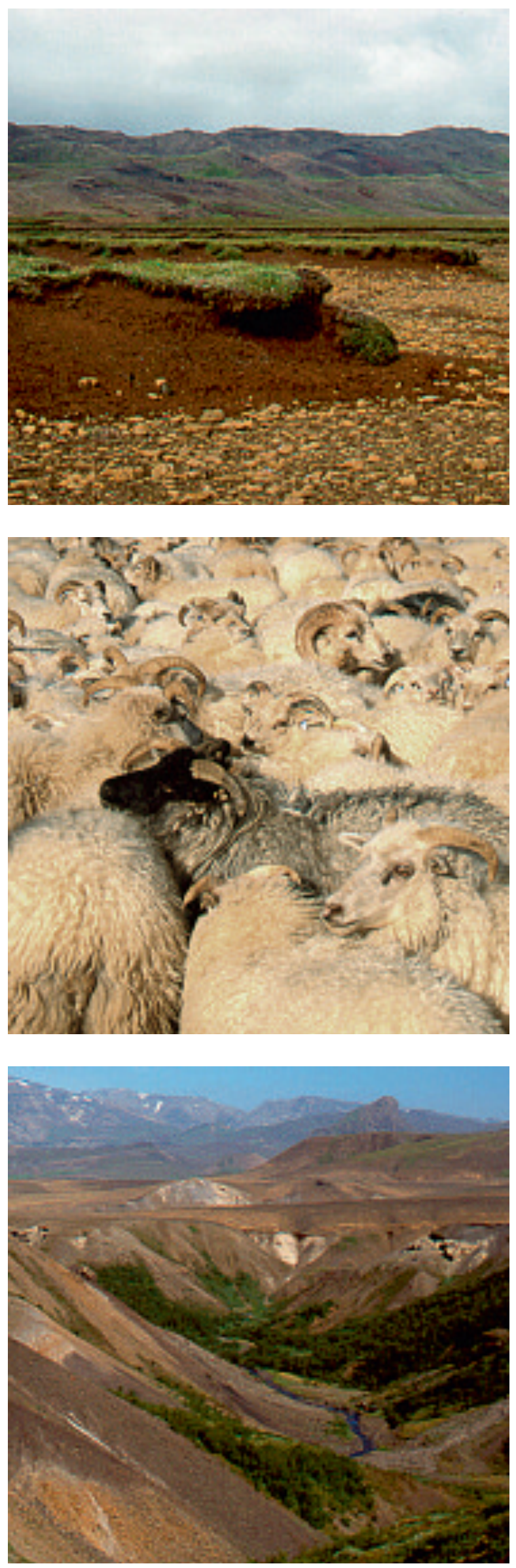

lige Streifenfarn (Asplenium trichomanes), das Sumpf-Labkraut (Galium palustre) oder die blaugrüne Vogesen-Rose (Rosa dumalis), die allesamt außerhalb Islands in Europa weit verbreitet sind.

Einige wenige Pflanzenarten der isländischen Flora tragen den Artbeinamen islandica bzw. islandia, obgleich sie nicht ausschließlich auf Island vorkommen. Hierzu gehört beispielsweise der Zwerg-Säuerling (Koenigia islandica), ein unscheinbares einjähriges Knöterichgewächs, das auf ganz Island verbreitet ist und eine der kleinsten Blütenpflanzen weltweit darstellt.

Die heutige Flora Islands trägt insgesamt überwiegend borealen Charakter (60-70\% der Sippen), obwohl Island i. d. R. nicht der borealen Landschaftszone zugeordnet wird (DiersSEN 1996). Die Borealis, die sich wie ein breites Band über die gesamte Nordhalbkugel zwischen dem 50. und 70. Breitengrad erstreckt, ist durch ein kalt gemäßigtes Klima und ausgedehnte Nadelwälder gekennzeichnet, die auf Island natürlicherweise fehlen. Die biogeographischen Verbreitungsmuster sprechen allerdings durchaus für eine Zuordnung zu dieser Vegetationszone. Etwa ein Drittel (30-40\%) der auf Island vorkommenden Arten sind arktisch-alpine, zumeist circumpolare Elemente, d. h. sie wachsen wie z. B. die Silberwurz (Dryas octopetala) in den Tundrengebieten der gesamten Nordhalbkugel sowie in den Alpen oberhalb der Waldgrenze. Manche sind allerdings nur arktisch verbreitet. Die übrigen Sippen Islands, zumeist Süßwasserpflanzen und Arten der Küsten, zeigen keine Zuordnung zu einem bestimmten Geoelement. Insgesamt ähnelt die Flora Islands aber überwiegend derjenigen Nordeuropas. 97\% der Arten Islands sind auch in Skandinavien anzutreffen und $87 \%$ auf den

Abb. 6 (oben): Starke Bodenerosion lässt pilzförmige Formen entstehen, an der die ehemalige Bodenmächtigkeit noch erkennbar ist.

Abb. 7 (Mitte): Zu viele Schafe mit der einhergehenden Beweidung tragen zur Bodenerosion bei.

Abb. 8 (unten): Ein kleines, stark geschütztes Waldstück. 
Echte endemische Pflanzensippen, die nur auf Island vorkommen, gibt es hingegen nur wenige. $\mathrm{Zu}$ erwähnen sind der Isländische Augentrost (Euphrasia calida) und die weißlich-rosablühende Unterart des arktischen Mohns (Papaver radicatum subsp. stefanssonii). Hinzu kommen zahlreiche besondere Pflanzenvarietäten, die sich nur in der Nähe heißer Quellen auf Island finden. Dazu gehört beispielsweise die Varietät fallax des Rippenfarns (Blechnum spicant), die im Gegensatz zu der normalen Form keine Unterscheidung zwischen fertilen und sterilen Wedeln aufweist. Ungeklärt ist derzeit noch der Einwanderungszeitpunkt dieser endemischen Varietäten. Es ist aber durchaus vorstellbar, dass diese besonderen Sippen bereits vor den Eiszeiten auf Island existierten und an ihren besonderen Standorten, eben den heißen Quellen, überdauerten.

Britischen Inseln, aber auch immerhin 66\% kommen in Grönland vor. Die Gemeinsamkeiten mit der nordamerikanischen Flora sind hingegen sehr gering. Nur neun Arten, darunter das Arktische Weidenröschen (Epilobium latifolium), die Westliche Waldhyazinthe (Platanthera hyperborea) oder die Westliche Saumnarbe (Lomatogonium rotatum) fehlen in Europa und haben ihr Verbreitungsgebiet außer auf Island nur in Nordamerika bzw. im Falle von Lomatogonium rotatum auch in Asien. Dies zeigt, dass Island nach den Eiszeiten nicht ausschließlich von Westen her besiedelt wurde. Als Transportmedien für Diasporen kommen Seevögel und andere Tiere, Meeres- und Luftströmungen und in jüngerer Zeit auch der Mensch in Frage.

Wie auch in anderen subarktischen und arktischen Regionen der Erde dominieren auf Island Seggen (8,7\%), Gräser (8,0\%), Kreuzblüter $(8,4 \%)$ sowie Korbblüter $(6,6 \%)$. $\mathrm{Zu}$ den artenreichsten Gattungen gehören Carex, Juncus und Salix. Innerhalb der Gattung Hieracium werden von einigen Autoren sogar mehr als 100 verschiedene Kleinarten für Island ausge- wiesen. Auch Orchideen (1,2\% aller Arten) muss man in Island nicht lange suchen. Immerhin ist diese Pflanzenfamilie mit acht Arten vertreten. Mitunter in großen Beständen tritt die bereits erwähnte Westliche Waldhyazinthe (Platanthera hyperborea) auf. Mit einer Höhe von $15-30 \mathrm{~cm}$ und kleinen, hellgrünen Blüten ist sie kleiner und unscheinbarer als die bei uns auf Trockenrasen verbreitete Grünliche Waldhyazinthe (P. chlorantha). Ähnlich unscheinbar sind Weißzüngel (Pseudorchis albida), Hohlzunge (Coeloglossum viridis) und das auf Island sehr seltene Zweiblatt (Listera ovata). Mit die schönste Orchidee Islands ist das Gefleckte Knabenkraut (Dactylorhiza maculata), das beispielsweise reichlich in Pórsmörk vorkommt. Als Lebensform überwiegen in der boreal-arktisch geprägten isländischen Flora Zwergsträucher und mehrjährige Stauden, einjährige Arten sind seltener, Geophyten so gut wie gar nicht anzutreffen.

\subsection{Floren- und Vegetationsgeschichte}

Um die Entwicklung der Flora und Vegetation Islands zu verstehen, muss man einen Blick zurück bis in das Tertiär werfen. Damals herrschte auf Island ein feucht-mildes Klima mit einer Jahresmitteltemperatur zwischen 10 und $15^{\circ} \mathrm{C}$ und es kam zur Bildung von Braunkohleflözen und Lateritböden. Die wärmeliebende arktotertiäre Flora bildete reiche Laub- und Laubmischwälder u.a. mit Fagus, Quercus, Acer, Ulmus, Platanus, Liriodendron, Ginkgo, Sassafras, Juglans, Magnolia, Laurus, Pinus, Picea, Larix, Metasequoia, Taxodium und Juniperus. Von ihnen sind zahlreiche Fossilien belegt. Damit zeigte die Flora Islands im Tertiär im Gegensatz zu heute eine große Ähnlichkeit sowohl zur nordamerikanischen als auch zur europäischen Flora. Es gilt heute als bewiesen, dass im Tertiär über die sog. Thule-Landbrücke ein intensiver Florenaustausch zwischen Nordamerika Grönland - Island - Färöer - Schottland und Europa stattgefunden hat. Diese Landbrücke bestand etwa bis vor zwei Mio. Jahren. Danach erst geriet Island in die jetzige isolierte Lage, die die Hauptursache für die heutige Artenarmut darstellt. Gegen Ende des Tertiärs kam es zu- 

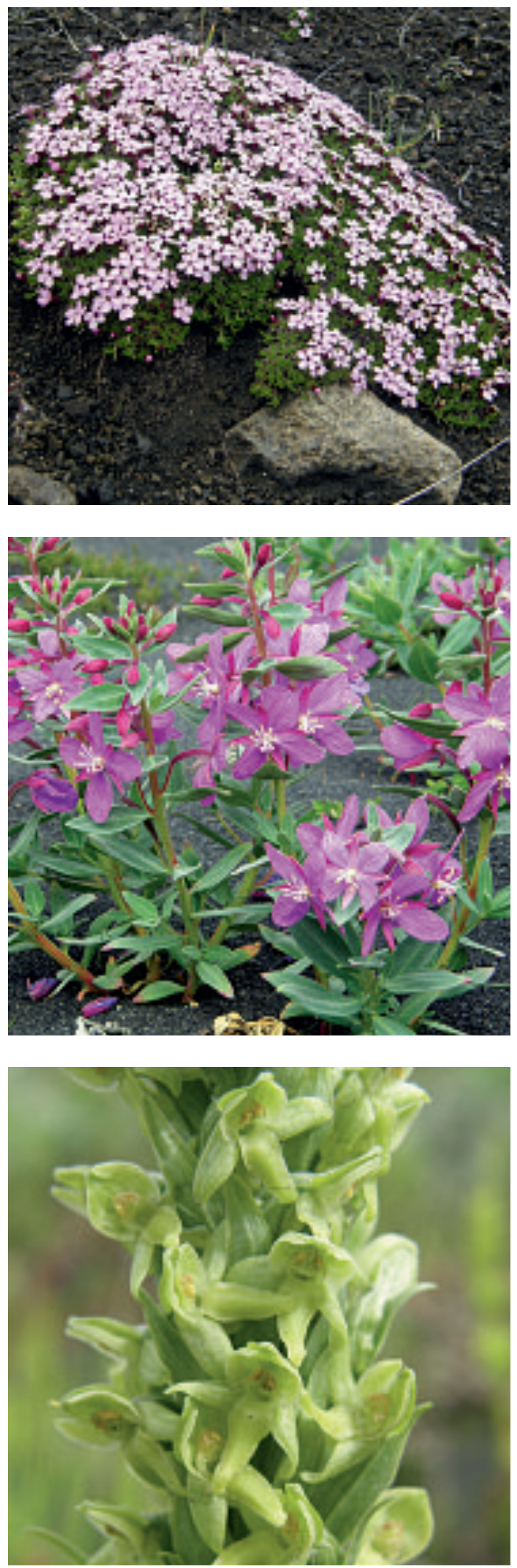

sätzlich zu einer deutlichen Abkühlung und damit zu einer allmählichen Zurückdrängung der thermophilen Laubwaldflora zugunsten einer borealen Nadelwaldflora. Ein weiterer Temperaturrückgang führte schließlich zu einer Auslöschung der Nadelbaumarten und einer Dominanz von Gehölzen der Gattungen Betula, Sorbus und Salix und leitete schließlich mit den ersten Vergletscherung höherer Gebirgsgipfel das Eiszeitalter (vor 3 Mio. - 10000 Jahren) ein. Wie überall auf der Welt war das Eiszeitalter auf Island durch den Wechsel von mehreren Kaltzeiten mit dazwischen liegenden Warmzeiten geprägt. Auch die postglaziale Wiedererwärmung war durch verschiedene wärmere und kühlere Phasen gekennzeichnet. Die Moorbirke (Betula pubescens) ist heute die auf Island zusammen mit der Eberesche (Sorbus aucuparia) und einigen Weidenarten einzige waldbildene Baumart. Unklar ist, ob sie wie in den nordskandinavischen Fjell-Regionen die subpolare Waldgrenze bei etwa 200-300 m bildet, die Eiszeiten auf Island überdauerte oder erst postglazial wieder einwanderte. Pollenanalysen belegen eine starke Wiederausbreitung der Birke vor 13000 Jahren in Schottland und vor 10000 Jahren in Süd-Island (HALlsdótTir \& CASELDINE 2003 ). Im Subboreal vor etwa 5000 Jahren lag die Jahresmitteltemperatur in Island sogar um $2-3{ }^{\circ} \mathrm{C}$ höher als heute. Weite Bereiche Islands waren mit Birkenwald bestanden und die Baumgrenze rückte auf $600 \mathrm{~m}$ über den Meeresspiegel. Danach wurde das Klima für die Vegetation wieder ungünstiger. Eine klimatische Gunstphase erlebten auch die ersten dauerhaften Siedler Islands. Als sich der erste norwegische Wikinger Ingólfur Arnarson 874 n. Chr. in Reykjavík niederließ und in den nachfolgenden Jahrzehnten eine große Einwanderungswelle über das Land rollte (sog. Landnahme-Zeit), war der ursprüngliche subpolare Birkenwald ebenfalls viel weiter verbreitet. Man

Abb. 9 (oben): Dichtes Polster von Silene acaulis, einem arktisch-alpinen Florenelement.

Abb. 10 (Mitte): Epilobium latifolium im Gletschervorfeld. Abb. 11 (unten): Platanthera hyperborea. 
Die Zahl der Neophyten stieg besonders sprunghaft nach dem 2. Weltkrieg an, als die internationalen Handelsbeziehungen des nun unabhängigen Island erweitert wurden. Wichtige Einwanderungstore für neue Pflanzen sind in Island insbesondere die Häfen sowie Baumschulen, in denen große Mengen an Jungpflanzen aus anderen Teilen der Erde bis zur Aufforstung kultiviert werden. In den mitgeführten Wurzelballen lassen sich im- mer wieder Diasporen fremder Sippen finden. Der Prozentsatz der eingebürgerten Sippen beträgt in der Flora von Island bereits $11 \%$, im Hauptstadtgebiet sogar bereits $20 \%$. Hinzu kommen unbeständige Sippen. Diese machen an der Gesamtflora einen Anteil von $27 \%$, im Gebiet von Reykjavík etwa $18 \%$ aus (Steinecke 1997). Somit weist die isländische Flora bereits einen sehr hohen Grad an Florenverfälschung auf. geht davon aus, dass etwa $24 \%$ der Landesfläche bewaldet waren. Das Klima war damals in Island so günstig, dass im Süden sogar der Anbau von Gerste und anderen Getreidearten möglich war. Heute ist dies in Island nicht mehr möglich, daher überwiegt in der Landwirtschaft die Viehwirtschaft. Folge der massiven Besiedlungswelle war eine bis in die heutige Zeit anhaltende Abnahme der ursprünglichen Waldbestände auf nur noch $1 \%$ der Gesamtfläche. Die Wälder litten insbesondere unter der Abholzung zur Gewinnung von Bau- und Brennholz und einer kontinuierlichen Überweidung durch die von den Wikingern mitgebrachten Haustiere, vor allem durch Schafe, die auch heute noch in einer Art Almwirtschaft gehalten werden. Daher konnten sich Reste der natürlichen Birkenwälder nur an abgeschiedenen und schwer zugänglichen Bereichen erhalten.

Von 1200 bis $1850 \mathrm{kam}$ es erneut zu einer Klimaveränderung. Es traten die geringsten Jahresmitteltemperaturen seit 8000 Jahren auf, nicht umsonst spricht man auch von der kleinen Eiszeit. Mit der zunehmenden Besiedlung Islands wurden nicht nur die ursprünglichen Lebensräume stark in Mitleidenschaft gezogen, sondern auch die einheimische Flora deutlich verändert. Die ersten Siedler führten bewusst und unbewusst neue Pflanzenarten nach Island ein, von denen sich viele etablierten. So brach-

Abb. 12: Neongrüne Moose prägen weite Landstriche im Hochland. ten die Wikinger Nutzplanzen wie Hordeum (als Getreide), Linum usitatissimum (zur Fasergewinnung) und Myrica gale, Artemisia absinthum (zur Herstellung von Met und als Heilkräuter) nach Island. Es folgten als Kulturfolger ruderale Pflanzenarten wie Alopecurus pratensis, Festuca pratensis, Trifolium pratense, die heute in Island etabliert und weit verbreitet sind.

Heute kommen zu den nativen Arten noch mehr als 250 nicht indigene Arten, und jedes Jahr werden er mehr.

Glücklicherweise sind unter den Neubürgern bisher aber nur wenige invasive Sippen, die die natürlichen Pflanzenbestände zurückdrängen. $\mathrm{Zu}$ erwähnen ist jedoch die Alaska-Lupine (Lupinus nootkatensis), die aufgrund ihrer stickstoffbindenen Fähigkeiten seit den 1980er

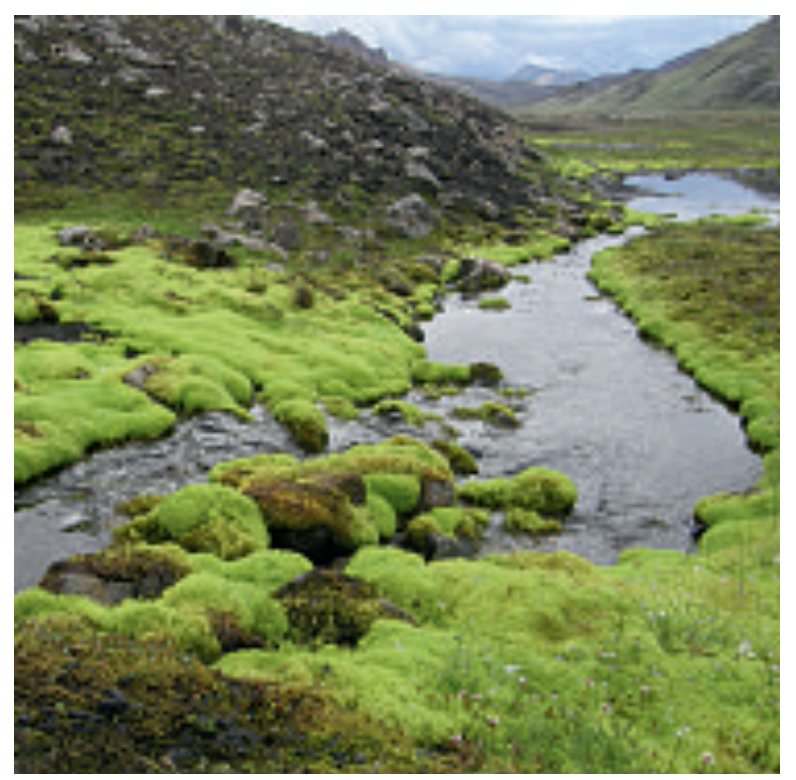



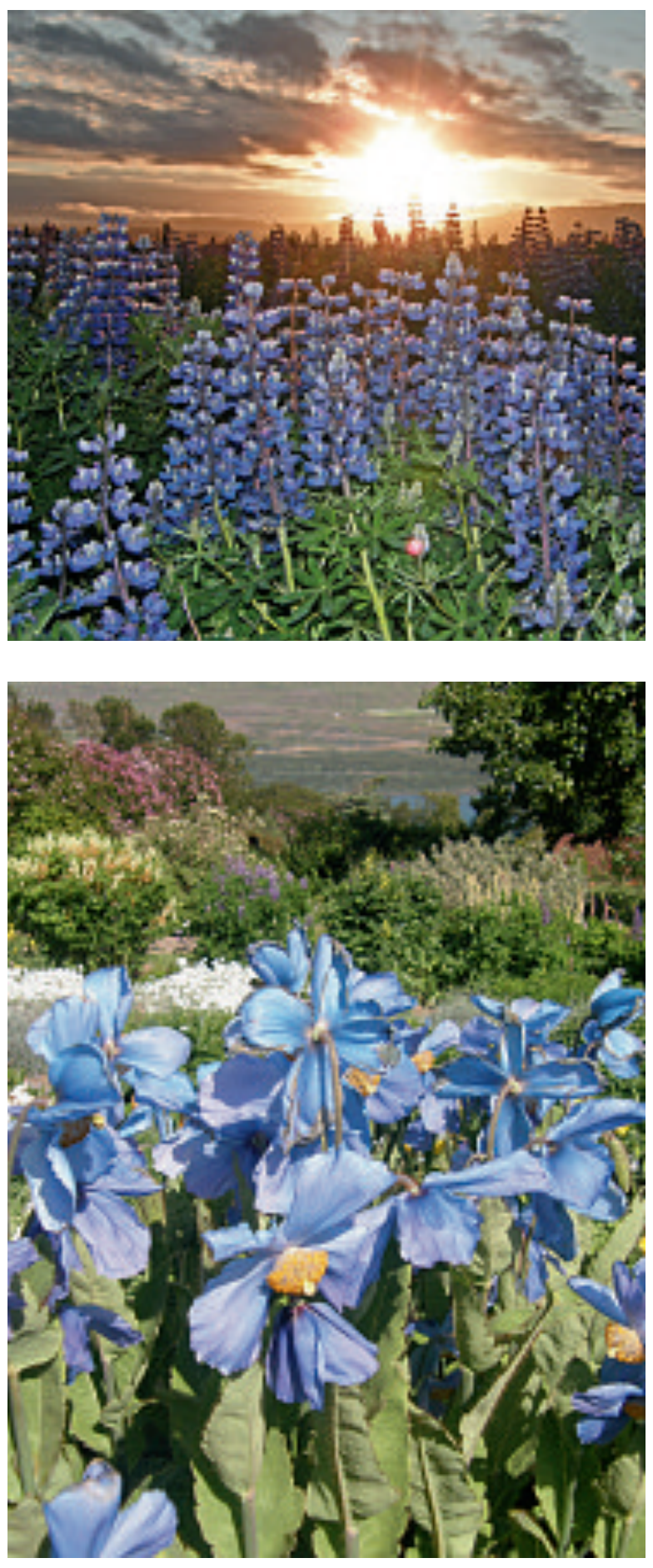

Jahren zur Bodenverbesserung und als Erosionsschutz auf vegetationsarmen Flächen gezielt angepflanzt wurde. Diese Art, obwohl sie wunderschön blau-weiß blüht, hat sich inzwischen so massiv vermehrt, dass einheimische, weniger wuchskräftige Vegetationsbestände stark zurückgedrängt werden und der ursprüngliche Landschaftscharakter verloren geht. Lediglich der Wiesenkerbel (Anthriscus sylvestris) scheint den Kampf mit der Lupine aufgenommen zu haben. Weiße Flächen durchmischen sich mit den blauen und es ist spannend, wie die heutigen Lupinenbestände in einigen Jahren aussehen werden.

Welche Pflanzenvielfalt theoretisch in Island gedeihen kann, beweisen die beiden botanischen Gärten Reykjavik im Südwesten und Akureyri im Norden Islands. Hier werden neben heimischen und alpinen Arten auch zahlreiche nordamerikanische und asiatische Prachtstauden kultiviert. Viel schöner als bei uns gedeiht dort aufgrund der hohen Luftfeuchtigkeit beispielsweise der Blaue Scheinmohn (Meconopsis grandis, M. betonicifolia).

\section{Literatur}

Buckland, P. C. \& Dugmore, A. J. 1991: The origins of the Icelandic biota in the light of recent research. - Environmental change in Iceland. - Dordrecht.

Dierssen, K. 1996: Vegetation Nordeuropas. - Stuttgart. EINARsson, E. 2005. Flóra og gróður Íslands, í bókinni Íslandsatlas. Edda útgáfa hf. í samstarfi við Fixlanda ehf. - Reykjavík.

Government of Canada (Hrsg.) 1996: Species at risk in Canada. - Ottawa.

Gudmundsson, A. T. \& Kjartansson, H. 1996: Land im Werden. Ein Abriss der Geologie Islands. - Reykjavik. Gudmundsson, A. T. 2007: Lebende Erde. Facetten der Geologie Islands. - Reykjavik.

Hallsdóttir, M. Caseldine C. J. 2003: The Holocene vegetation history of Iceland, state-of-the-art and future research. - Palaeogeogr., Palaeoclimat., Palaeoecol. 193: $535-560$

Nordal, J. \& Kristinsson, V. (Hrsg.) 1996: Iceland. The republic. Handbook published by the Central Bank of Iceland. - Reykjavík.

Sснміdт, F.-U. 1991: Ísland. Naturkundlicher Reiseführer Nr. 1. - Göttingen.

Schutzbach, W. 1999: Island. Feuerinsel am Polarkreis. - Bonn.

Steinecke, K. 1997: Stadtflora und Stadtvegetation in Reykjavík. - Norden 12: 157-180.

\section{Internetseiten}

http://www.ni.is/grodur/valisti/

The Icelandic Institute of Natural History (Hrsg.) 2010

http://www.welt-in-zahlen.de/index.phtml

Abb. 13 (oben): Lupinus nootkatensis, ein Neophyt aus Nordamerika, der schnell große Flächen besiedeln kann.

Abb. 14 (unten): Im botanischen Garten Akureyri gedeihen verschiedene Meconopsis-Arten sehr üppig. 